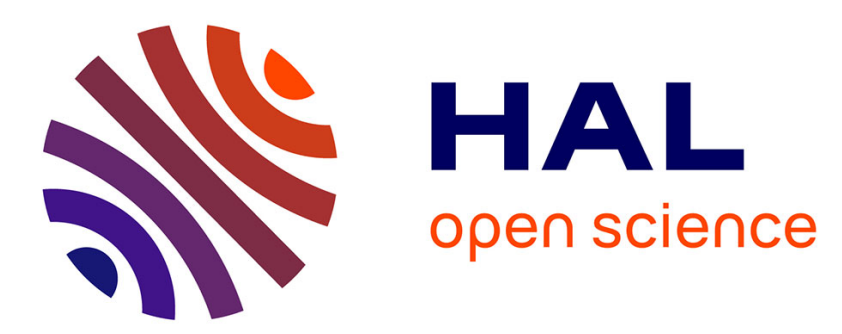

\title{
A humanoid walking pattern generator for sole design optimization
}

\author{
Adrien Pajon, Giovanni de Magistris, Sylvain Miossec, Kenji Kaneko, \\ Abderrahmane Kheddar
}

\section{- To cite this version:}

Adrien Pajon, Giovanni de Magistris, Sylvain Miossec, Kenji Kaneko, Abderrahmane Kheddar. A humanoid walking pattern generator for sole design optimization. ICAR 2015 - 17th International Conference on Advanced Robotics, Jul 2015, Istanbul, Turkey. pp.105-110, 10.1109/ICAR.2015.7251441 . hal-01278857

\section{HAL Id: hal-01278857 \\ https://hal.science/hal-01278857}

Submitted on 1 Mar 2016

HAL is a multi-disciplinary open access archive for the deposit and dissemination of scientific research documents, whether they are published or not. The documents may come from teaching and research institutions in France or abroad, or from public or private research centers.
L'archive ouverte pluridisciplinaire HAL, est destinée au dépôt et à la diffusion de documents scientifiques de niveau recherche, publiés ou non, émanant des établissements d'enseignement et de recherche français ou étrangers, des laboratoires publics ou privés. 


\title{
A Humanoid Walking Pattern Generator for Sole Design Optimization
}

\author{
Adrien Pajon ${ }^{3,1}$, Giovanni De Magistris ${ }^{3,1}$, Sylvain Miossec ${ }^{2}$, Kenji Kaneko ${ }^{1}$ and Abderrahmane Kheddar ${ }^{1,3}$
}

\begin{abstract}
We devised a new walking pattern generator based on a minimization of the energy consumption and that offers the necessary parameters to be used in flexible sole design problems. This pattern generator is implemented in two sets of experiments implying the HRP-2 humanoid robot: generating walking pattern generator with different weighting between (i) the center of mass forces, and (ii) torques applied at the ankle joint; we also considered two types of feet: the HRP-2 built-in flexible ankle, and our proposed solution that keeps the ankleto-foot without flexibility and add instead a flexible sole. The latter shows several benefits that are discussed.
\end{abstract}

\section{INTRODUCTION}

Bipedal walking on regular grounds is a well-documented and thoroughly studied problem in the humanoid robotics research. An excellent synthesized review is presented in [1]. Although an adequate level of robustness and maturity is reached in designing bipedal walking, much work remains to be done in particular for walking on uneven terrains.

Recent studies show this maturity level for humanoid walking on flat floor. For example, walking with human-inloop [2], walking with human stylized gaits [3][4] or walking with emotional behavior gaits [5]. In the latter works, the precise control at the interface between the humanoid robot and the sole (ground) plays an important role. For example, reducing impacts and having smooth walking is critical in human-in-the-loop walking under hard visual servoing constraints [6] or for precise stylized or emotional walking gaits. To reduce the impact at the landing, different studies showed the importance of the foot shape design [7][8].

Comparing humanoid robots to humans, an effort must be made to design the soles to compensate as much as possible: (i) the limited number of degree-of-freedom in current humanoid robots; (ii) their rigid mechanical design unlike the compliance found in human joints and links; and (iii) the robust (implicit) control of the feet-ground interaction (contact). In fact, a well-designed sole may include as much as possible the missing ingredients as far as their effects may be projected in the contact operational space. Therefore, human joint/link compliance effect has a projected equivalence in the operational space that can be embedded with the sole design. Since walking and its tuning matters the implicit control of the feet/ground (contact) interaction, it is worthwhile addressing the sole design (for a given feet) with a particular interest.

However, in order to design the sole as a shape optimization problem, integrating existing walking pattern generators

\footnotetext{
${ }^{1}$ CNRS-AIST Joint Robotics Laboratory (JRL), UMI3218/CRT, Japan.

${ }^{2}$ PRISME Laboratory, University of Orléans, Bourges 18020, France.

${ }^{3}$ CNRS-University of Montpellier, Laboratoire d'Informatique de Robotique et de Microélectronique de Montpellier, IDH, France.
}

(PG) is not straightforward. Since we model the sole deformation in quasi-static, the orientation of the foot is directly linked to the ZMP of the foot and the sole's shape. Among other technical reasons required by the use of flexible soles (i) we must manage a ZMP under each foot, especially in the double support phase, and (ii) we must have a smooth ZMP to get a smooth foot orientation. To date, no existing PG fulfills such requirements. We then present a PG accounting for the latter issues which is formulated as a QP with an energy criteria. We assess our PG with walking experiments involving the HRP-2 robot having either ankle flexibility or simple flexible soles.

\section{WALKING PATTERN GENERATOR}

To formulate a PG to be used in a sole optimization problem, coherent choices are to be made for: (i) the model used, which allows to compute the criteria from the parameters, (ii) the criteria to optimize, and (iii) the parameters defining in which space optimal solutions are to be searched.

There are several works that formulate walking gaits as optimization problems, e.g. [9][10][11]. Almost all use an inverted pendulum model with a point mass (1). Wieber et al. [9] compute smooth zero moment point (ZMP) and center of mass (COM) trajectories by minimizing the jerk of the COM. In [11] a similar criterion is used, but the number of steps and walking speed that minimize energy consumption are accounted. The approach in [10] generates stable walking gaits by bringing the ZMP close to ankle positions.

Walking is split in two phases: (i) a single support phase (SSP), with one foot on the floor, and (ii) a double support phase (DSP), where both feet touch the floor. During the DSP, we note with subscript ' 1 ' the foot that leaves the floor at the end of DSP, and with subscript ' 2 ', the foot that comes in contact at the beginning of DSP.

\section{A. Simplified/reduced mechanical model}

Using the cart-table model in Fig. 1b proposed in [10], the ZMP position expression on $x$-axis is:

$$
x_{\mathrm{ZMP}}=x_{\mathrm{COM}}-\frac{z_{\mathrm{COM}}}{g} \ddot{x}_{\mathrm{COM}}
$$

The computation on $x$-axis can easily be extended to $y$-axis.

\section{B. Forces}

Using the model in Fig. 1a and considering the leg mass negligible w.r.t COM, the static mechanical equilibrium is given by two opposite and equal forces: the COM force and floor reaction force (ZMP force), that is:

$$
\mathbf{f}_{\mathrm{zMP}}(t)=-\mathbf{f}_{\text {Сом }}(t)
$$




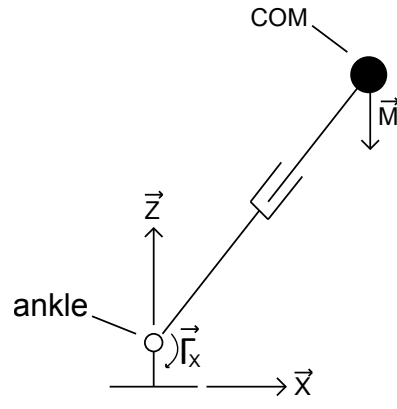

(a) Simplified robot model (b) Cart-table model

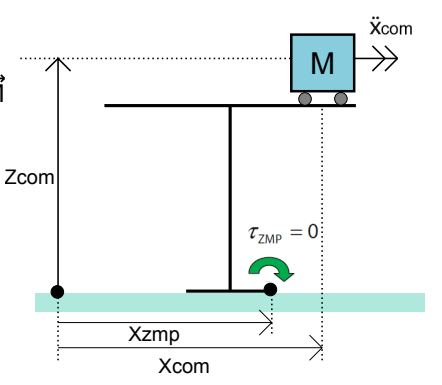

Fig. 1: Reduced model in sagittal plane and cart-table model

Imposing COM position at constant height during the walking gait, the COM vertical acceleration is zero. Subsequently, the COM force can be expressed as:

$$
\mathbf{f}_{\text {Сом }}(t)=m\left(\ddot{\mathbf{P}}_{\text {сом }}(t)+g \vec{z}\right)
$$

where $\ddot{\mathbf{P}}_{\text {Сом }}$ is the COM acceleration.

\section{Torques}

Considering only the foot in contact and assuming it is at a fixed position during the whole SSP, we have:

$$
\boldsymbol{\Gamma}_{a}(t)=\mathbf{f}_{\mathrm{ZMP}}(t) \times\left(\mathbf{P}_{a}(t)-\mathbf{P}_{\mathrm{ZMP}}(t)\right),
$$

subscript $a$ stands for the ankle joint, $\boldsymbol{\Gamma}_{a}$ is the torque at the ankle, $\mathbf{P}_{a}$ is the ankle position and $\mathbf{P}_{\mathrm{ZMP}}$ is the ZMP position.

During the DSP, the two feet are in contact. Considering independently each foot and assuming that they are at a fixed position during the whole DSP, we have:

$$
\begin{aligned}
& \boldsymbol{\Gamma}_{a_{1}}(t)=\mathbf{f}_{\mathrm{ZMP}_{1}}(t) \times\left(\mathbf{P}_{a_{1}}(t)-\mathbf{P}_{\mathrm{ZMP}_{1}}(t)\right) \\
& \boldsymbol{\Gamma}_{a_{2}}(t)=\mathbf{f}_{\mathrm{ZMP}_{2}}(t) \times\left(\mathbf{P}_{a_{2}}(t)-\mathbf{P}_{\mathrm{ZMP}_{2}}(t)\right)
\end{aligned}
$$

\section{Optimization criteria}

We chose our criteria to be quadratic and as close as possible to energy consumption, while using the simple pendulum model of Fig. 1b given by (1). From [11], energy consumption taking into account motor and reducer model has the following expression form:

$$
E=\sum_{j} \int_{t_{i}}^{t_{f}}\left(a_{j} \tau_{j}^{2}+b_{j} \tau_{j} \dot{q}_{j}+c_{j} \dot{q}_{j}^{2}\right) d t
$$

where $\tau_{j}$ is force/torque and $\dot{q}_{j}$ is the joint $j$ velocity, and $a_{j}, b_{j}, c_{j}$ are coefficients depending on joint $j$ motor and reducer. Using the simplified robot model of Fig. 1a, (6) becomes:

$$
E=\int_{t_{i}}^{t_{f}}\left(a_{a} \Gamma_{a}^{2}+b_{a} \Gamma_{a} \dot{\theta}+c_{a} \dot{\theta}^{2}+a_{p} f_{p}^{2}+b_{p} f_{p} \dot{r}+c_{p} \dot{r}^{2}\right) d t
$$

where subscripts $p$ is for a prismatic joint, $\dot{\theta}$ is the ankle velocity, $f_{p}$ is the prismatic force, and $\dot{r}$ is the prismatic velocity.

However, except $\Gamma_{a}$, the $\dot{\theta}, f_{p}$ and $\dot{r}$ are nonlinear functions of the COM and the ZMP. To keep a quadratic criteria, only the term $\Gamma_{a}^{2}$ is kept. The term $f_{p}^{2}$ is approximated with
$\mathbf{f}_{\mathrm{COM}}^{2}$, where $\mathbf{f}_{\mathrm{COM}}$ is the force acting on the COM. Finally for a $3 \mathrm{D}$ pendulum, $\Gamma_{a}^{2}$ is replaced by $\left\|\boldsymbol{\Gamma}_{a}(t)\right\|^{2}$ and $\mathbf{f}_{\mathrm{COM}}^{2}$ by $\left\|\mathbf{f}_{\text {сом }}(t)\right\|^{2}$, and we end up with the criterion:

$$
\mathcal{C}=\min \left\{\int_{t_{i}}^{t_{f}}\left(\lambda\left\|\mathbf{f}_{\text {сом }}(t)\right\|^{2}+\mu\left\|\boldsymbol{\Gamma}_{a}(t)\right\|^{2}\right) d t\right\}
$$

where $\lambda$ and $\mu$ are the weights of each criteria, $\mu=1-\lambda$ and $(\lambda, \mu) \in[0,1]$. Since motor characteristics are not known for the reduced model used, we did not know how to choose these two weights. Therefore, experiments on a real robot determine the weights so that our criterion is the best to minimize total walking energy consumption.

This criteria is interesting since by taking $\lambda=1$ we obtained a walking pattern close to [9][11]; while taking $\lambda=0$ we found a solution with ZMP close to ankle like in [10]. Intermediate values of $\lambda$ give compromises between the two approaches.

\section{ZMP AND COM TRAJECTORIES}

To define the movement of the simplified biped robots in Fig. 1a, we computed optimized ZMP and COM trajectories. We specifically computed ZMP trajectories under each foot during the DSP, based on the study of the force distribution. This aspect is not commonly studied in the literature. Our formulation of the sole optimization problem requires knowing ZMP trajectories under each foot during the DSP.

To compute the ZMP and COM trajectories, we used the cart-table model (1), where the $x_{\text {сом }}$ trajectory is defined as a $5^{\text {th }}$ order polynomial function (minimum jerk invariant theory). Based on [12] and [13], modeling the ZMP trajectories by polynomials of a given degree $\alpha$ and solving (1), result in a COM trajectory with the polynomial part having the same order $\alpha$. Therefore, we define the ZMP trajectory as a $5^{\text {th }}$ order polynomial. Since the orientation of the foot is directly linked to the ZMP position, we also have a smooth variation of the foot's orientation.

\section{A. ZMP trajectories}

The walking is defined in three sequences:

1) Starting sequence: the robot begins with a DSP. This sequence starts on the point $\mathrm{ZMP}_{\text {initial }}$.

2) Walking sequences: the robot does $n$ steps. Each step is a sequence of 'half SSP' $\rightarrow$ 'DSP' $\rightarrow$ 'half SSP'.

3) Stopping sequence: the robot ends with a DSP. This sequence ends on the point $\mathrm{ZMP}_{\text {final }}$.

Each walking sequence is defined as two 'half SSP' and one 'DSP'. Four via-points are defined to parametrize each duration of these phases, see Fig. 2. For a given walking sequence $q$, we have:

- The first 'half SSP' goes from via-point $\mathrm{A}_{q}$ to $\mathrm{B}_{q}$.

- The DSP' goes from via-point $\mathrm{B}_{q}$ to $\mathrm{C}_{q}$.

- The last 'half SSP' goes from via-point $\mathrm{C}_{q}$ to $\mathrm{D}_{q}$.

Based on these walking gait sequences, we need $m=$ $3 n+2$ polynomials to model the whole ZMP trajectory, each of which writes:

$$
x_{\mathrm{ZMP}}^{(j)}(t)=\sum_{i=0}^{5} a_{i}^{(j)}\left(\Delta t_{j}\right)^{i}
$$




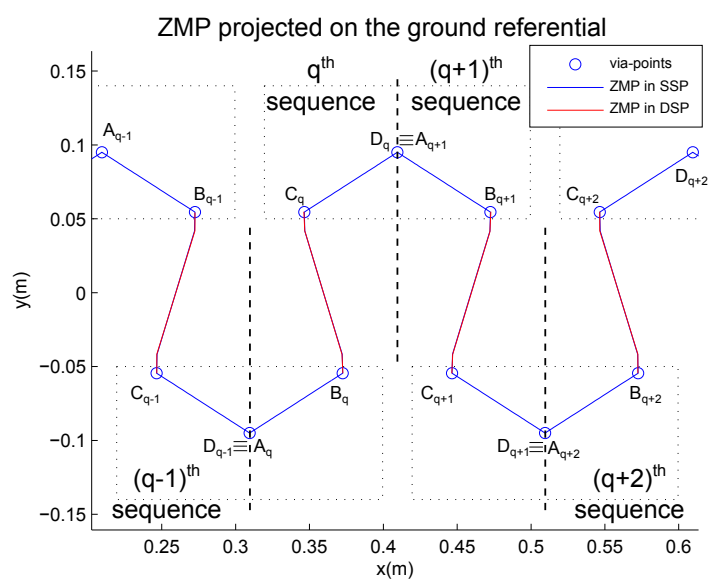

Fig. 2: Example of walking sequences with via-points (o) and ZMP trajectories during SSP (blue) and DSP (red)

where the superscript $j$ denotes the $j^{\text {th }}$ phases and $a_{i}$ is the $i^{\text {th }}$ polynomial coefficient. In (9) $\Delta t_{j}=t-t_{j-1}$, where $t_{j-1}$ is the ending time of phase $j-1$.

To find the polynomial coefficients of (9) and obtain smoother trajectories, we enforced the boundary conditions in position, speed and acceleration at each via-point.

To obtain the polynomial functions of the ZMP trajectory, we interpolated them between $\mathrm{ZMP}_{\text {initial }} \rightarrow \mathrm{C}_{1}$ during starting sequence, $\mathrm{A}_{q} \rightarrow \mathrm{B}_{q}, \mathrm{~B}_{q} \rightarrow \mathrm{C}_{q}$ and $\mathrm{C}_{q} \rightarrow \mathrm{D}_{q}$ during the sequence $q$ and $\mathrm{B}_{n+2} \rightarrow \mathrm{ZMP}_{\text {final }}$ during stopping sequence. In our ZMP trajectory definition, $\mathrm{C}_{1}$ is equal to $\mathrm{A}_{2}, \mathrm{D}_{q}$ to $\mathrm{A}_{q+1}$ and $\mathrm{D}_{n+1}$ to $\mathrm{B}_{n+2}$. $\mathrm{ZMP}_{\text {initial }}$ and $\mathrm{ZMP}_{\text {final }}$ are not necessarily equal to $\mathrm{B}_{1}$ and $\mathrm{C}_{n+2}$ respectively.

To define $\mathrm{ZMP}_{1}$ and $\mathrm{ZMP}_{2}$ trajectories, we added the viapoints $\mathrm{B}_{q}^{\prime}$ and $\mathrm{C}_{q}^{\prime}$ assigned to both trajectories respectively. To find these polynomials, we interpolated them between $\mathrm{B}_{q} \rightarrow \mathrm{B}_{q}^{\prime}$ and between $\mathrm{C}_{q}^{\prime} \rightarrow \mathrm{C}_{q}$.

Using the ZMP definition, we obtain:

$$
\boldsymbol{\Gamma}_{\mathrm{ZMP}}^{(j)}\left(\mathbf{f}_{\mathrm{ZMP}_{1}}\right)+\boldsymbol{\Gamma}_{\mathrm{ZMP}}^{(j)}\left(\mathbf{f}_{\mathrm{ZMP}_{2}}\right)=0
$$

where $\boldsymbol{\Gamma}_{\mathrm{ZMP}}^{(j)}\left(\mathbf{f}_{\mathrm{ZMP}_{i}}\right)$ is the torque of the reaction force $\mathbf{f}_{\mathrm{ZMP}_{i}}$ under foot $i \in\{1,2\}$ applied on ZMP during the phase $j$.

Solving (10) in the direction $\vec{y}$ we can write:

$$
\sum_{i=1}^{2}\left(x_{\mathrm{ZMP} i}^{(j)}-x_{\mathrm{ZMP}}^{(j)}\right) f_{\mathrm{ZMP}_{i}}^{z(j)}=0
$$

where $x_{\mathrm{ZMP}}$ is the component $x$ of the ZMP position and $f_{\mathrm{ZMP}_{i}}^{z}$ is the component $z$ of the reaction force under the foot $i \in\{1,2\}$.

From (11), we obtain the following relationship between $\mathrm{ZMP}_{1}$ and $\mathrm{ZMP}_{2}$ :

$$
x_{\mathrm{ZMP}_{2}}^{(j)}=x_{\mathrm{ZMP}}^{(j)}-\frac{f_{\mathrm{ZMP}}^{z(j)}}{f_{\mathrm{ZMP}_{2}}^{z(j)}}\left(x_{\mathrm{ZMP}_{1}}^{(j)}-x_{\mathrm{ZMP}}^{(j)}\right)
$$

From (12), if $\mathrm{ZMP}_{1}$ trajectories and the repartition between $f_{\mathrm{ZMP}_{1}}^{z}$ and $f_{\mathrm{ZMP}_{2}}^{z}$ are known, we do not need to interpolate polynomials to obtain $\mathrm{ZMP}_{2}$ trajectories.
Based on the Fig. 1a's model and related hypotheses, the relationship between $f_{\mathrm{ZMP}_{1}}^{z}$ and $f_{\mathrm{ZMP}_{2}}^{z}$ can be found:

$$
\begin{aligned}
& f_{\mathrm{ZMP}}^{z(j)}+f_{\mathrm{ZMP} 2}^{z(j)}=f_{\mathrm{ZMP}}^{z(j)}=m g \\
& f_{\mathrm{ZMP}_{1}}^{z(j)}=m g-f_{\mathrm{ZMP}_{2}}^{z(j)}
\end{aligned}
$$

where the reaction force $f_{\mathrm{ZMP}_{2}}^{z}$ in (13) is defined by:

$$
f_{\mathrm{ZMP} 2}^{z(j)}=k^{(j)} \cdot f_{\mathrm{ZMP}}^{z(j)}=k^{(j)} \cdot m g
$$

$k$ in (14) is a $5^{\text {th }}$ order polynomial representing the repartition of ZMP force under each foot.

From (12), (13) and (14), we obtain:

$$
x_{\mathrm{ZMP}_{2}}^{(j)}=x_{\mathrm{ZMP} 1}^{(j)}-\frac{1}{k^{(j)}}\left(x_{\mathrm{ZMP}_{1}}^{(j)}-x_{\mathrm{ZMP}}^{(j)}\right)
$$

The boundary conditions at the start of the DSP during a walking cycle are:

$$
\begin{aligned}
& f_{\mathrm{ZMP} 2}^{z(j)}=0, f_{\mathrm{ZMP} 1}^{z(j)}=f_{\mathrm{ZMP}}^{z(j)}=m g, \\
& x_{\mathrm{ZMP} 1}^{(j)}=x_{\mathrm{ZMP}}^{(j)}=x_{B}^{(j)}, \dot{x}_{\mathrm{ZMP} 1}^{(j)}=\dot{x}_{\mathrm{ZMP}}^{(j)}=\dot{x}_{B}^{(j)}, \\
& \ddot{x}_{\mathrm{ZMP} 1}^{(j)}=\ddot{x}_{\mathrm{ZMP}}^{(j)}=\ddot{x}_{B}^{(j)} \text { and } x_{\mathrm{ZMP} 2}^{(j)} \neq x_{B}^{(j)}
\end{aligned}
$$

Deriving (11) and (16), we obtain:

$$
\left[\begin{array}{c}
f_{\mathrm{ZMP}}^{z(j)} \\
\dot{f}_{\mathrm{ZMP} 1}^{z(j)} \\
\ddot{f}_{\mathrm{ZMP} 1}^{z(j)}
\end{array}\right]=\left[\begin{array}{c}
m g \\
0 \\
0
\end{array}\right] \text { and }\left[\begin{array}{c}
f_{\mathrm{ZP}_{2}}^{z(j)} \\
\dot{f}_{\mathrm{ZMP} 2}^{z(j)} \\
\ddot{f}_{\mathrm{ZMP} 2}^{z(j)}
\end{array}\right]=\left[\begin{array}{l}
0 \\
0 \\
0
\end{array}\right]
$$

Similarly, we obtain for the end of the DSP:

$$
\left[\begin{array}{c}
f_{\mathrm{ZMP}}^{z(j)} \\
\dot{f}_{\mathrm{ZMP}}^{z(j)} \\
\ddot{f}_{\mathrm{ZMP} 1}^{z(j)}
\end{array}\right]=\left[\begin{array}{l}
0 \\
0 \\
0
\end{array}\right] \text { and }\left[\begin{array}{c}
f_{\mathrm{ZMP} 2}^{z(j)} \\
\dot{f}_{\mathrm{ZMP}}^{z(j)} \\
\ddot{\mathrm{Z}}_{\mathrm{ZMP} 2}^{z(j)}
\end{array}\right]=\left[\begin{array}{c}
m g \\
0 \\
0
\end{array}\right]
$$

Equations (17) and (18) represent the initial and final conditions to interpolate $k$ in (15). $k$ is a function which varies from 0 to 1 .

During the starting and the stopping sequences, to obtain a robust optimization algorithm w.r.t to the different walking parameters, we added new via-points to $\mathrm{ZMP}_{1}$ and $\mathrm{ZMP}_{2}$ trajectories. In particular, we added two via-points: $\mathrm{B}_{q}^{\prime \prime}$ between $\mathrm{B}_{q} \rightarrow \mathrm{B}_{q}^{\prime}$ and $\mathrm{C}_{q}^{\prime \prime}$ between $\mathrm{C}_{q} \rightarrow \mathrm{C}_{q}^{\prime}$. On these new via-points, $\mathrm{ZMP}_{1}$ and $\mathrm{ZMP}_{2}$ trajectories fulfill the boundary conditions in position, speed and acceleration.

To define the function $k$, we took into consideration experimental studies on human walking [14]. During the starting sequence, humans move their COM laterally to be under the stance limb (we noted foot 2). The starting sequence begins with an equal repartition of load in each limb (under each foot in our case). Then, the lateral movement is induced by a momentary load of the swing limb (we noted foot 1). To finish, the stance limb is fully loaded and the swing limb is unloaded to begin the first SSP. For this reason, we chose the function $k$ equal to 0.5 on $\mathrm{C}_{1}^{\prime}, 0.25$ on $\mathrm{C}_{1}^{\prime \prime}$ and 1 on $\mathrm{C}_{1}$.

The human behavior to stop walking is symmetrical to the starting sequence [14]. During the stopping sequence, humans move laterally their COM and decelerate their movement. This lateral movement begins with a full load of the stance limb (foot 1) from the end of the SSP. Then, the deceleration is induced by a momentary load of the swing 
limb (foot 2). The stopping sequence ends with the same load repartition on each limb (under each foot in our case) [14]. For this reason, we chose the function $k$ equal to 0 on $\mathrm{B}_{n+2}$, 0.75 on $\mathrm{B}_{n+2}^{\prime \prime}$ and 0.5 on $\mathrm{B}_{n+2}^{\prime}$.

\section{B. COM trajectories}

Based on Morisawa et al. [13], (1) can be rewritten as:

$$
\ddot{x}_{\mathrm{COM}}^{(j)}=\frac{g}{z_{\mathrm{COM}}}\left(x_{\mathrm{COM}}^{(j)}-x_{\mathrm{ZMP}}^{(j)}\right)
$$

Substituting (9) into (19), the COM position can be expressed by:

$$
x_{\mathrm{COM}}^{(j)}=V^{(j)} c_{j}+W^{(j)} s_{j}+\sum_{i=0}^{5} A_{i}^{(j)}\left(\Delta t_{j}\right)
$$

where:

$$
\begin{aligned}
& c_{j}=\cosh \left(\omega_{j} \Delta t_{j}\right) \\
& s_{j}=\sinh \left(\omega_{j} \Delta t_{j}\right) \\
& \omega_{j}=\sqrt{\frac{g}{z_{\mathrm{COM}}^{(j)}}}=\sqrt{g / z_{\mathrm{COM}}} \\
& A_{i}^{(j)}= \begin{cases}a_{i}^{(j)}+\sum_{k=1}^{(5-i) / 2} b_{i+2 k}^{(j)} a_{i+2 k}^{(j)} & \text { for } i=0 \ldots 3 \\
a_{i}^{(j)} & \text { for } i=4,5\end{cases} \\
& b_{i+2 k}^{(j)}=\prod_{l=1}^{k} \frac{(i+2 l)(i+2 l-1)}{w_{j}^{2}}
\end{aligned}
$$

$V^{(j)}$ and $W^{(j)}$ are the unknowns of the system. In (20), $a_{i}^{(j)}$ coefficients are known. This is the difference between (20) and the system of equations in [13].

Equation (20) has $2 m$ unknowns with $m=3 n+2$ phases. These unknowns satisfy the following boundary conditions for the COM position and velocity:

1) Initial

$$
\begin{aligned}
& x^{(1)}\left(T_{0}\right)=V^{(1)}+A_{0}^{(1)} \\
& \dot{x}^{(1)}\left(T_{0}\right)=W^{(1)}+A_{1}^{(1)}
\end{aligned}
$$

2) Relationship between two successive sequences

$$
\begin{aligned}
& V W^{(j)}+\sum_{i=0}^{5} A_{i}^{(j)}\left(\Delta T_{j}\right)^{i}=V^{(j+1)}+A_{0}^{(j+1)} \\
& V W \omega^{(j)}+\sum_{i=1}^{5} i A_{i}^{(j)}\left(\Delta T_{j}\right)^{i-1}=W^{(j+1)} \omega_{j}+A_{1}^{(j+1)}
\end{aligned}
$$

where

$$
\begin{aligned}
& V W^{(j)}=V^{(j)} C_{j}+W^{(j)} S_{j} \\
& V W \omega^{(j)}=V^{(j)} \omega_{j} S_{j}+W^{(j)} \omega_{j} C_{j} \\
& C_{j}=\cosh \left(\omega_{j} \Delta T_{j}\right), \quad S_{j}=\sinh \left(\omega_{j} \Delta T_{j}\right)
\end{aligned}
$$

3) Final

$$
\begin{aligned}
& x^{(m)}\left(T_{m}\right)=V W^{(m)}+\sum_{i=0}^{5} A_{i}^{(m)}\left(\Delta T_{m}\right)^{i} \\
& \frac{d x^{(m)}}{d t}\left(T_{m}\right)=V W \omega^{(m)}+\sum_{i=1}^{5} i A_{i}^{(m)}\left(\Delta T_{m}\right)^{i-1}
\end{aligned}
$$

where $\Delta T_{j}=t_{j}-t_{j-1}$.

From the boundary conditions (21)-(25), the total conditions are $2 m+2$. Removing COM velocity conditions on initial and final phases (they are solved at the pattern optimization level), $2 m$ conditions remain. The unknowns can be calculated then by the following system:

$$
\mathbf{G} \cdot \mathbf{y}=\mathbf{N} \cdot \mathbf{x}+\mathbf{H} \cdot \mathbf{l}
$$

where

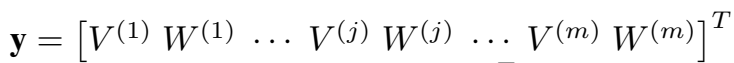

$$
\begin{aligned}
& \mathbf{x}=\left[\begin{array}{llllll}
x_{\mathrm{COM}}^{(1)}\left(T_{1}\right) & 0 & \cdots & 0 & x_{\mathrm{COM}}^{(m)}\left(T_{m}\right)
\end{array}\right]^{T} \\
& \mathbf{l}=\left[\begin{array}{lllllll}
A_{0}^{(1)} & \cdots & A_{5}^{(1)} & \cdots & A_{0}^{(m)} & \cdots & A_{5}^{(m)}
\end{array}\right]^{T} \\
& \mathbf{G}_{h, q}= \begin{cases}{\left[\begin{array}{ll}
1 & 0
\end{array}\right]} & \text { for }(h, q)=(1,1) \\
{\left[C_{m} S_{m}\right]} & \text { for }(h, q)=(m+2, m) \\
G_{1, h} & \text { for } q=h-1 \\
G_{2, h} & \text { for } q=h \\
0 & \text { otherwise }\end{cases} \\
& \mathbf{N}_{h, q}= \begin{cases}1 & \forall(h, q) \in\{(1,1) ;(2 m, 2 m)\} \\
0 & \text { otherwise }\end{cases}
\end{aligned}
$$

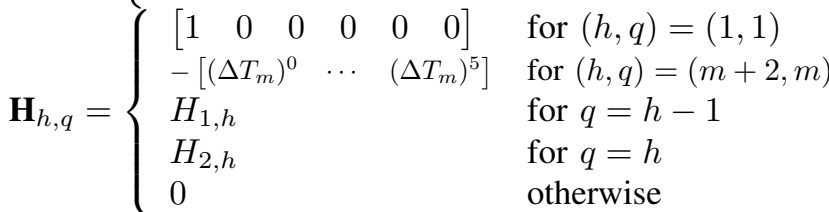

with $h=1, \ldots, m+2, q=1, \ldots, m$,

$$
\begin{aligned}
\mathbf{G}_{1, h} & =\left[\begin{array}{ccc}
C_{j} & S_{h} \\
\omega_{h} S_{h} & \omega_{h} C_{h}
\end{array}\right], \mathbf{G}_{2, h}=\left[\begin{array}{cc}
-1 & 0 \\
0 & -\omega_{h}
\end{array}\right], \\
\mathbf{H}_{1, h} & =-\left[\begin{array}{cccc}
\left(\Delta T_{m}\right)^{0} & \left(\Delta T_{m}\right)^{1} & \cdots & \left(\Delta T_{m}\right)^{5} \\
0 & \left(\Delta T_{m}\right)^{0} & \cdots & \left(\Delta T_{m}\right)^{4}
\end{array}\right], \\
\mathbf{H}_{2, h} & =\left[\begin{array}{llllll}
1 & 0 & 0 & 0 & 0 & 0 \\
0 & 1 & 0 & 0 & 0 & 0
\end{array}\right] .
\end{aligned}
$$

\section{Optimization parameters}

To obtain the ZMP and COM trajectories, we used a QP optimization based on the cost function (8). The optimization parameters are:

- Position, speed and acceleration at via-points of ZMP $(9 n+3$ parameters $)$

- Feet positions ( $n-1$ parameters)

- Position, speed and acceleration at via-points of $\mathrm{ZMP}_{1}$ $(3 n+12$ parameters $)$

We imposed:

- Initial and final feet positions

- Initial and final position, speed and acceleration of ZMP

- Durations of the SSP and the DSP

- The COM height

To enforce the humanoid stability during walking, the ZMP trajectories must be inside the support convex hull [15][16]. The support convex hull is defined by the area of the support foot. To obtain this area, we added inequality constraints to our PG optimization problem. During the SSP, this condition is linear and can be integrated as inequality constraints into a QP. During DSP, this condition is not linear. However, if $\mathrm{ZMP}_{1}$ and $\mathrm{ZMP}_{2}$ trajectories are in the support convex hull defined by the areas of each foot, the stability condition for ZMP trajectories is fulfilled. These conditions are linear and can also be integrated in the QP optimization.

Based on human walking results [17] and to compensate the robot precision [10], we defined a feet support areas with a security margin of $5-10 \%$ of the foot length. To avoid auto-collisions, we defined a minimal distance between the robot feet $(3 \mathrm{~cm})$. To avoid stretched legs singularity, we 
chose a maximum step length $(30 \mathrm{~cm})$. To respect the boundary condition for the COM position and velocity, we added the equality constraints (22) and (26) to the optimization.

\section{RESULTS}

Now we present and analyze the results of our PG. We implemented an tested our obtained walking motion in experiments conducted with the HRP-2 robot [18]: first with the built-in feet including an ankle flexibility, then with our specially designed feet with flexible sole.

For this study, we considered only straight line walking for $1 \mathrm{~m}$ with 10 steps to test the PG on the robot. This is enough to have none-biased results and to assess the PG before its use (future work) in a sole optimization problem.

\section{A. Pattern optimization results}

The Fig. 3a shows part of the obtained ZMP and COM reference trajectories for different values of $\lambda$ :

- $\lambda \rightarrow$ 1: minimization of COM force only

- $\lambda \rightarrow 0$ : minimization of ankle torque only

- $\lambda=0.5$ : minimization with equal weights on COM force and on ankle torque

As expected, for $\lambda=0$ we obtain ZMP and COM trajectories similar to [12][10] with large lateral variations of the COM. For $\lambda \rightarrow 1$ trajectories are similar to [9][19] with small lateral variations of the COM and with a ZMP close to the feet edge. The mid-compromise solution $\lambda=0.5$ gives limited lateral variations of the COM with a ZMP similar to that of a human walk [14]. All solutions result in walk with right and left feet as close as possible, so as to minimize lateral variations of $\mathrm{COM}$ (and hence $\mathrm{COM}$ force) and to minimize variation of ZMP in DSP. We did not consider the case $\lambda=1$ since it is not well-posed: COM force criteria does not depend on ZMP of each foot in the DSP which could then be any trajectory in the feet.

The Fig. $3 \mathrm{~b}$ shows $\mathrm{ZMP}_{1}$ and $\mathrm{ZMP}_{2}$ trajectories during the DSP. Both $\mathrm{ZMP}_{2}$ and $\mathrm{ZMP}_{1}$ go from toe to heel. Considering only one foot contact, this gives a toe-heel-toeheel ZMP trajectory, which is different from the human heeltoe ZMP trajectory [20]. This result is a direct consequence of minimizing ankle torque with our reduced model: indeed, for the landing foot it is better that the ZMP goes through the foot's center to minimize the ankle torque than staying in the heel (same reasoning stands for take-off foot). For a flexible sole this means first foot contact is on the toe, then the foot rotates around the heel, the toe and the heel again. We believe that taking into account foot rotation with a flexible sole in our future work, will allow to write a better ankle energy consumption criterion that would penalize this behavior.

\section{B. Experiments with ankle flexibilities}

We tested our walking patterns on the HRP-2 humanoid robot, see the accompanying video. Each time we compared with the 'test' pattern from [13].

Fig. 4 shows the evolution of the torques in the right foot ankle (results are similar to the left part). Theoretical ankle
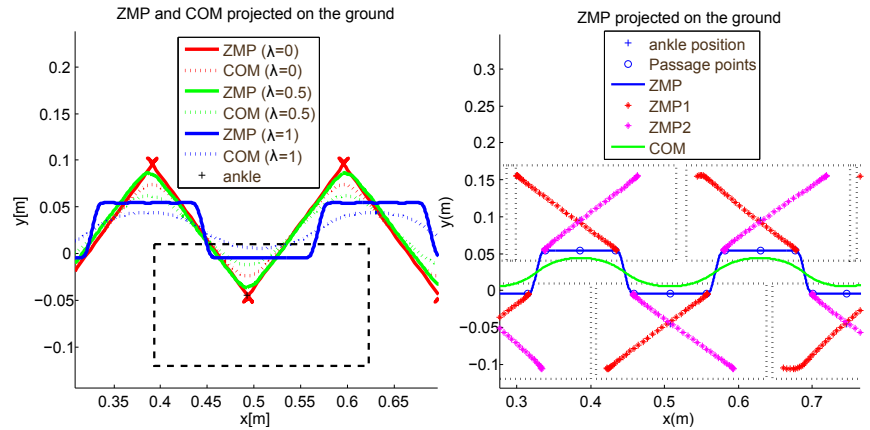

(a) Different values of $\lambda$ and $\mu$

(b) ZMP
0.01 )

Fig. 3: ZMP and COM trajectories

torques increase with $\lambda$ since the weight associated to the ankle in the torque criterion is decreased. More precisely:

- From $\lambda=0$ to 0.2 , torques are larger than the theoretical ones. This might be due to the difficulty to compensate for the flexibility deformation;

- From $\lambda=0.3$ to 0.5 , torques are close to theoretical ones;

- From $\lambda=0.6$ to 0.99 , torques are larger than the theoretical ones. Indeed, important deformation happens when ZMP is close to the edge of the foot, which is a difficult situation to stabilize.

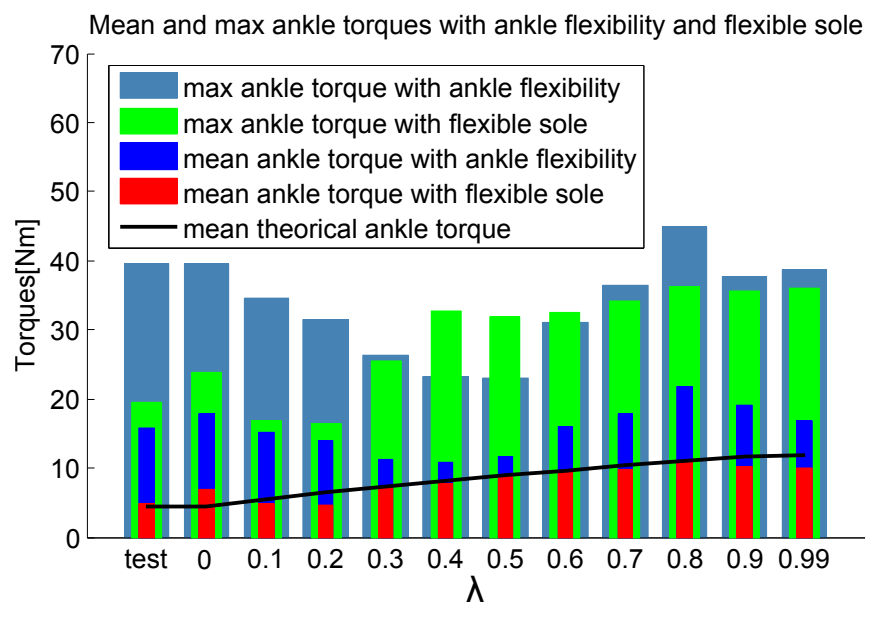

Fig. 4: Ankle torque with flexible soles and ankle flexibilities

Fig. 5 shows the evolution of the COM acceleration in the lateral direction (which is also the image of force on $\mathrm{COM}$ ). We do not show the COM acceleration in the walking direction as it is almost not varying with $\lambda$. Theoretical COM acceleration decreases with $\lambda$ since the ankle's weight in the torque criterion is increased:

- From $\lambda=0$ to 0.5 , the evolution of COM acceleration is similar to the theoretical one, yet with additional variations, that might be due to the stabilization;

- From $\lambda=0.6$ to 0.99 , as for torques in ankle, the COM acceleration is not decreasing because of the instability when the ZMP is close to the foot's edge. 


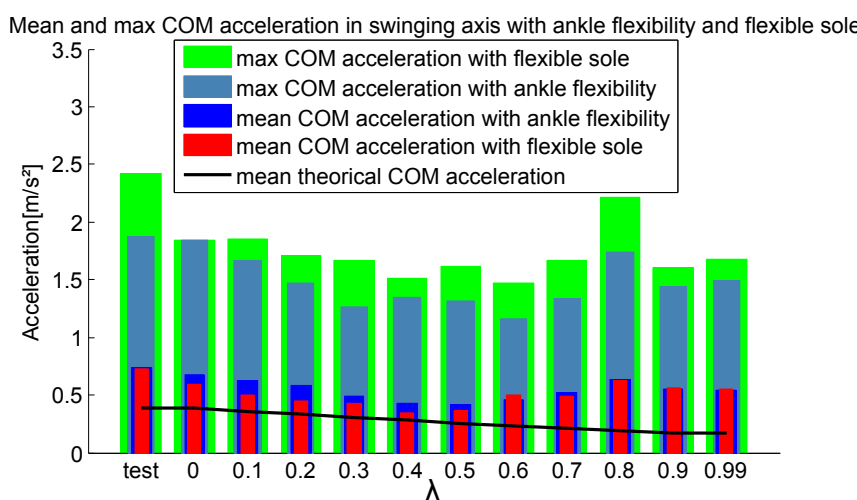

Fig. 5: COM acceleration in the swinging direction with flexible soles and ankle flexibilities

The energy consumption for the whole walk can be estimated by:

$$
E=\int_{t_{i}}^{t_{f}}\left(\sum_{j} s_{j}(t) \cdot \Gamma_{j}(t)\right) d t
$$

where $s_{j}$ is the actuator's angular velocity $j$ and $\Gamma_{j}$ its torque.

Fig. 6 shows the evolution of the energy needed by the left leg of the robot to achieve the whole walk (it is similar for the right leg). We observe a minimum in the energy consumption for $\lambda=0.2,0.3$, which is the best compromise between our criteria. In this case we obtain a $15 \%$ decrease of energy consumption compared to [13].

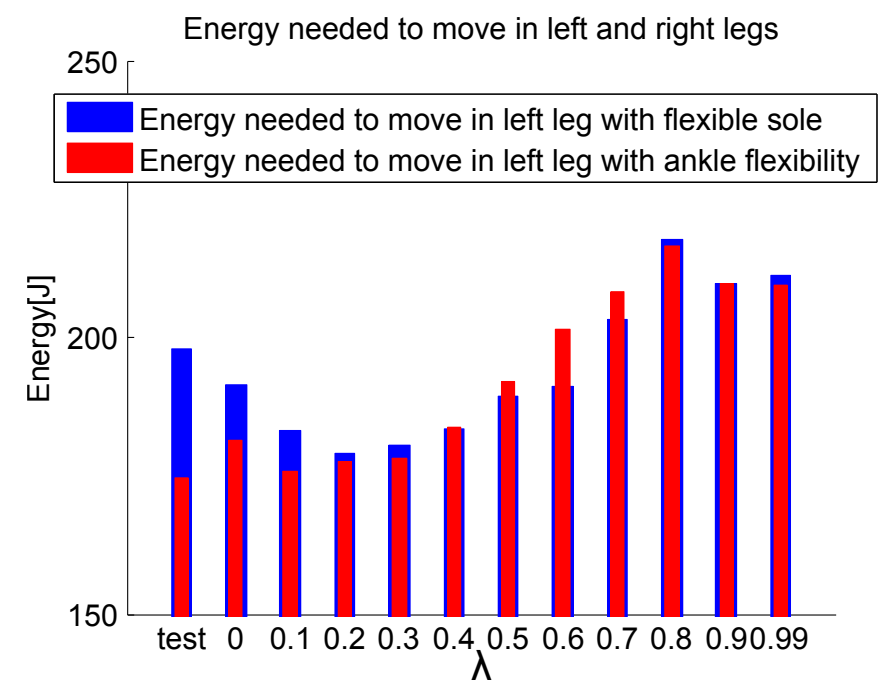

Fig. 6: Energy needed by left leg to achieve the whole walk

\section{Experiment with flexible sole}

We did the same experiments as previously with different feet of the HRP-2, see accompanying video. We removed the springs in ankle and added a flexible sole to absorb the landing impact shock. Fig. 7 shows HRP-2's new feet.

Fig. 4 shows the evolution of torques in right foot ankle with new feet (results are similar in left foot ankle). For all $\lambda$, evolution of experimental torques is similar to the theoretical
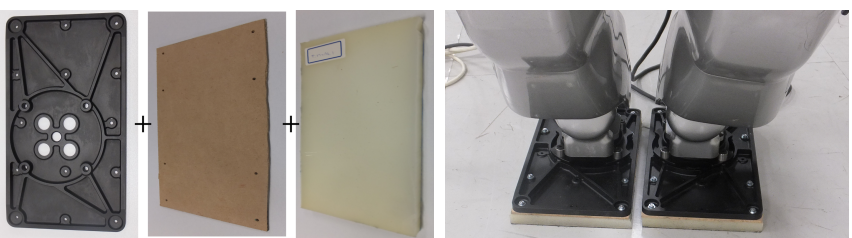

Fig. 7: Photo of HRP-2's feet with flexible soles

ones, even for $\lambda$ from 0.6 to 0.99 , when the walk becomes unstable (see video). In comparison to previous ankle flexible foot, the mean values of torques in ankle with new feet have almost been reduced by $5 \mathrm{Nm}$.

However, for lateral COM acceleration of Fig. 5, mean COM acceleration with new feet is still more than the theoretical one, but is slightly better than ankle flexible foot.

Additionally, some instability can be observed in the video with new feet when the ZMP is close to the foot edge, while with old feet it is more stable. In future work, we shall improve the sole flexibility stabilization control scheme that was tuned for the old feet system. We also hope to reduce part of this instability by soldes design.

We also looked at the impact force during walking: when walk is stable, maximum force is about $650 \mathrm{~N}$ for old feet and $630 \mathrm{~N}$ for new feet, while for unstable walk it can reach $700 \mathrm{~N}$ for both types of feet.

The minimum energy consumption for $\lambda=0.2,0.3$ is still valid for new feet with flexible soles, as can be observed in Fig. 6.

\section{CONCLUSION}

We devised a PG based on the minimization of energy consumption simplified by the minimization of COM forces and torques at the ankle level. Our PG computes smooth ZMP and COM trajectories from $5^{\text {th }}$-order polynomials and also generates ZMP under each foot during the double support phase (DSP). Using this definition of ZMP under each foot, it is possible to check the stability conditions in the DSP and to write them in a linear form. In addition, this formulation is useful for a quasi-static sole deformation model as the orientation of the foot is directly linked to these parameters. Thus, it will allow to compute human-like foot rolling depending on the sole shape design.

HRP-2's experiments showed that the flexibility in the ankles (due to the built-in shock-absorbing mechanism) induces relatively high torques. Without these springs, torques in ankle were reduced. Therefore, as an alternative to each ankle shock-absorbing mechanism to protect the robot structure and improve its stability during walking, we can now consider using flexible soles.

The experimental comparison between the latter options, revealed the effects of ZMP and COM trajectories on the walking motion and impacts on the robots. Indeed, by choosing the weights of the optimization criterion, it was possible to tune the walking gait at will.

As shown in IV-A, the ZMP under each foot has a toeheel-toe-heel trajectory. In a future work, we will study a 
third criteria to integrate in our cost function to have a heeltoe human like ZMP trajectory under each foot.

Now we are ready to follow-up this study by (i) integrating our proposed PG to the sole design optimization, (ii) build the optimized sole, and finally (iii) test the optimized sole on HRP-2 and HRP-4 humanoid robots.

\section{REFERENCES}

[1] P.-B. Wieber, S. Kuindersma, and R. Tedrake, "Modeling and control of legged robots," in Springer Handbook of Robotics, 2nd ed., B. Siciliano and O. Khatib, Eds. Springer Berlin Heidelberg, 2015.

[2] A. Bussy, A. Kheddar, A. Crosnier, and F. Keith, "Human-humanoid haptic joint object transportation case study," in IEEE/RSJ International Conference on Intelligent Robots and Systems, Vilamoura, Portugal, 7-12 October 2012, pp. 3633-3638.

[3] K. Miura, M. Morisawa, S. Nakaoka, F. Kanehiro, K. Harada, K. Kaneko, and S. Kajita, "Robot motion remix based on motion capture data -towards human-like locomotion of humanoid robots," in IEEE/RAS International conference on Humanoid Robots, Paris, France, December 7-10 2009, pp. 596-603.

[4] K. Mombaur, A. Truong, and J.-P. Laumond, "From human to humanoid locomotion- an invese optimal control approach," Autonomous Robot, vol. 28, pp. 369-383, 2010.

[5] M. L. Felis, K. Mombaur, H. Kadone, and A. Berthoz, "Modeling and identification of emotional aspects of locomotion," Journal of Computational Science, vol. 4, pp. 255-261, 2013.

[6] D. J. Agravante, A. Cherubini, A. Bussy, P. Gergondet, and A. Kheddar, "Collaborative human-humanoid carrying using vision and haptic sensing," in IEEE International Conference on Robotics and Automation. Hong Kong, China: IEEE, 31 May - 7 June 2014, pp. 607-612.

[7] P. Adamczyk, S. Collins, and A. Kuo, "The advantages of a rolling foot in human walking.” J Exp Biol., vol. 209 (Pt 20), pp. 3953-3963, october 2006.

[8] S. Miyakoshi and G. Cheng, "Examining human walking characteristics with a telescopic compass-like biped walker model," in IEEE International Conference on Systems, Man and Cybernetics, 2004, pp. $1538-1543$.

[9] A. Herdt, H. Diedam, P.-B. Wieber, D. Dimitrov, K. Mombaur, and M. Diehl, "Online walking motion generation with automatic foot step placement," Advanced Robotics, vol. 24, no. 5-6, pp. 719-737, 2010.

[10] S. Kajita and B. Espiau, Handbook of Robotics. Springer-Verlag, 2008, ch. Legged Robots, pp. 361-389.

[11] H.-K. Shin and B. K. Kim, "Energy-efficient gait planning and control for biped robots utilizing the allowable zmp region," Robotics, IEEE Transactions on, vol. 30, no. 4, pp. 986-993, August 2014.

[12] K. Harada, S. Kajita, K. Kaneko, and H. Hirukawa, "An analytical method on real-time gait planning for a humanoid robot," International Journal of Humanoid Robotics, october 82004.

[13] M. Morisawa, K. Harada, S. Kajita, K. Kaneko, F. Kanehiro, K. Fujiwara, S. Nakaoka, and H. Hirukawa, "A biped pattern generation allowing immediate modification of foot placement in real-time," in IEEE-RAS International Conference on Humanoid Robots, 2006, pp. $581-586$.

[14] D. A. Winter, "Human balance and posture control during standing and walking," Gait and Posture, vol. 3, pp. 193-214, December 1995.

[15] M. Vukabratovic and D. Juricic, "Contribution to the synthesis of biped gait," IEEE Transactions on Bio-Medical Engineering, vol. BME-16, no. 1, January 1969.

[16] M. Vukabratovic and B. Borovac, International Journal of Humanoid Robotics, vol. 1, no. 1, pp. 157-173, January 2004.

[17] M. B. Popovic, A. Goswani, and H. Herr, "Ground reference points in legged locomotion : definitions, biological trajectories and control implications," International Journal of Robotics Research, vol. 24, no. 12, pp. 1013-1032, December 2005.

[18] K. Kaneko, F. Kanehiro, S. Kajita, H. Hirukawa, T. Kawasaki, M. Hirata, K. Akachi, and T. Isozumi, "Humanoid robot hrp-2," in Proceedings of IEEE International Conference on Robotics and Automation, New Orleans, USA, April 2004, pp. 1083-1090.

[19] P.-B. Wieber, "Trajectory free linear model predictive control for stable walking in the presence of strong perturbations," in IEEE-RAS International Conference on Humanoid Robots, 2006, pp. 137 - 142.

[20] C. L. Vaughan, B. L. Davis, and J. C. O'Connor, Dynamics of human gait, C. Vaughan, Ed. Kiboho, 1992. 